\title{
Application of 3D Laser Scanner to Special-Shaped Curved Curtain Wall Construction
}

\author{
Cao Yajun, Che Zhiyan, Liu Cipei \\ China Construction Shenzhen Decoration Co., Ltd, Shenzhen, China \\ Email address: \\ 123722413@qq.com (Cao Yajun),7959025@qq.com (Che Zhiyan),402258871@qq.com (Liu Cipei)
}

To cite this article:

Cao Yajun, Che Zhiyan, Liu Cipei. Application of 3D Laser Scanner to Special-Shaped Curved Curtain Wall Construction. Engineering and Applied Sciences. Vol. 5, No. 6, 2020, pp. 114-118. doi: 10.11648/j.eas.20200506.13

Received: October 21, 2020; Accepted: November 18, 2020; Published: December 22, 2020

\begin{abstract}
With China's economic development and improvement of Chinese people's living quality in recent years, Chinese people have new requirements for innovative and fashionable buildings. Therefore, there are many fully special-shape and curved buildings that are measured with conventional curtain wall and are time-consuming with heavy workload emerging in endlessly. These special-shape buildings can be measured with a 3D laser scanner that is applicable to overall large-scope measurement and re-measurement, can directly shoot and scan the set scope so as to reduce measurement workload, and can be directly bound to BIM. In comparison with conventional measurement, 3D laser scanner has great advantages in special-shape curtain wall construction. 3D laser scanner has been applied to China Building Science and Technology Museum Project, CSCEC Star of Optical Valley for measurement and remeasurement and been highly recognized by the Designer, the Employer and other parties in convenience, high efficiency and other aspects. In consideration of high efficiency, convenience and bonding to BIM of 3D laser scanner in China Building Science and Technology Museum Project, can shorten the construction period and reduce the labor input, it is believed that $3 \mathrm{D}$ laser scanner will be the preferred measurement measure for special-shape curved curtain wall construction in the future and will be widely promoted and applied in the field and create greater value.
\end{abstract}

Keywords: 3D Laser Scanner, Special-shaped Curve, BIM, Curtain Wall Construction

\section{Background for Applicable of 3D Laser Scanner to Curtain Wall Construction}

China Building Science and Technology Museum is located in Wuhan City, Hubei, China, with a building area of $190000 \mathrm{~m}^{2}$. It is designed by Mr. Li Zuyuan, a famous architect in Taiwan. It aims to inherit Chinese architectural culture, promote the development of building science and technology, build a cultural communication platform with the greatest influence in the fields of Chinese architectural culture, exhibition and popular science, and become the image display window of Chinese Architectural Science and technology and architectural culture [1]. China Building Science and Technology Museum, CSCEC Star of Optical Valley, has a seed shape with curtain wall height of $58.13 \mathrm{~m}$. Its external facade is special-shape hyperboloid. The curtain wall system includes hyperbolic stainless steel plate curtain wall system, hyperbolic ball glass curtain wall system, and hyperbolic blade glass curtain wall system. It is hard to accurately lay off with conventional CAD software due to unique shape and different size, dimension and interval of panels on the external facade. Therefore, Laying-off shall be based on BIM model [2]. As the project duration is limited, the requirement for duration of follow-up works fails to be satisfied based on conventional BIM model. As a result, a new way is required for rapidly building BIM model so as to guide laying-off of subsequent materials and field construction [3]. 3D laser scanner is found through data collection in many aspects and is applied to measurement of special-shape curved curtain wall in combination with its application cases.

The equipment can be directly used for fully automatic dot scan of scanning area set, can quickly obtain 3D data (spatial point and location information) of the surface of measured objects, and can quickly present actual data on site through high-speed laser scanning so as to generate point cloud through bundled software and form models quickly [4]. As laying-off of subsequent materials is 
directly influenced by measurement, attention needs to be paid to proper use of 3D laser scanner [4].

\section{Use of 3D Laser Scanner}

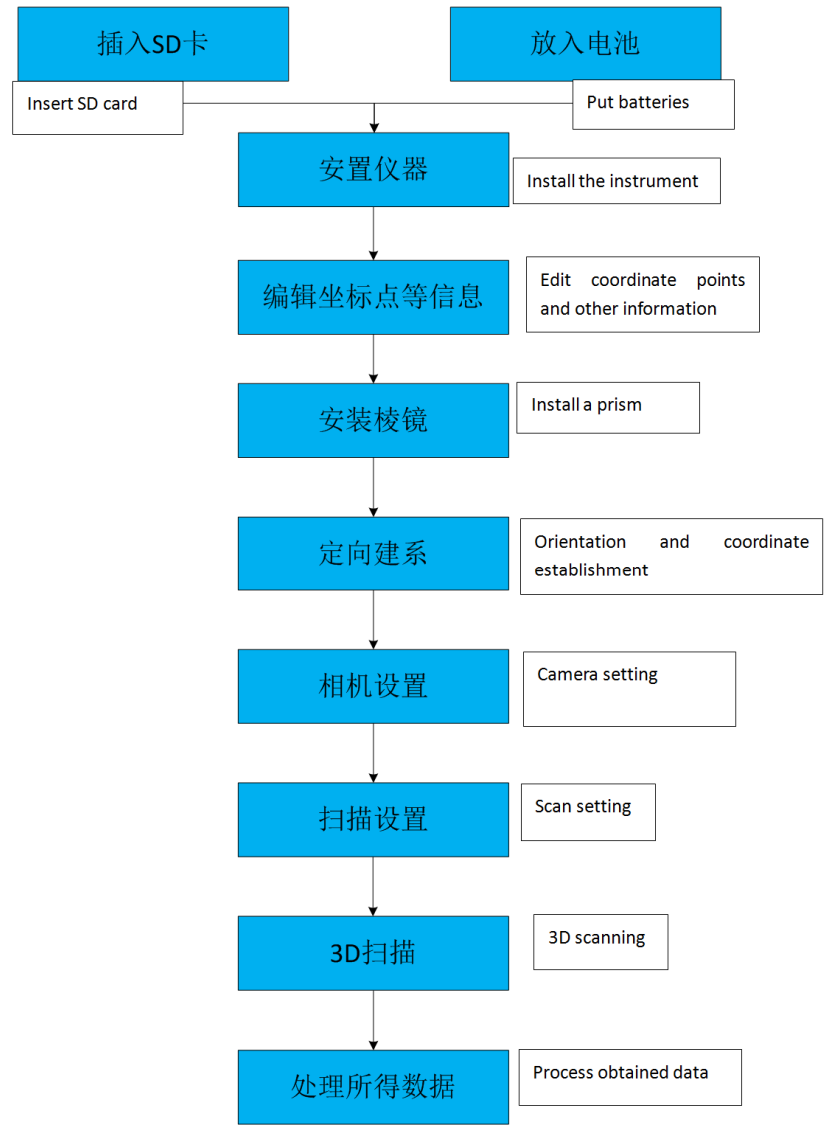

Figure 1. Work-flow of 3D Laser Scanner.

\subsection{Instrument Installation}

\subsubsection{Place a Tripod}

Firstly, stretch legs of the tripod to proper height; then, fasten screws in the middle-upper part of legs of the tripod.

\subsubsection{Place the Instrument}

Place the instrument on the tripod; fasten central screws; fix the instrument. Slightly loosen central screws [5]; move the instrument on the top of the tripod to make the instrument center directly above the measured point; fasten central screws.

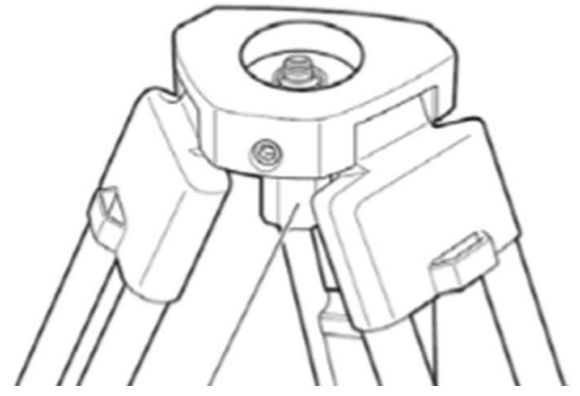

Figure 2. Central screws.

\subsubsection{Leveling}

Screw the two foot screws A and B so as to move the round bubble to the straight line perpendicular to center lines of the two foot screws [6].

Then, screw the foot screw $\mathrm{C}$ to make the round bubble in the middle.

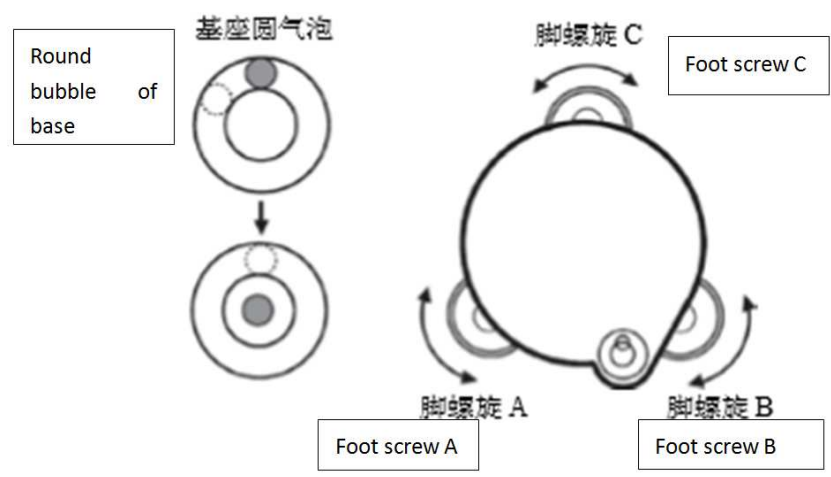

Figure 3. Leveling (1).

Use laser plummet for centering: Turn on power switch and click the tilt setting key.

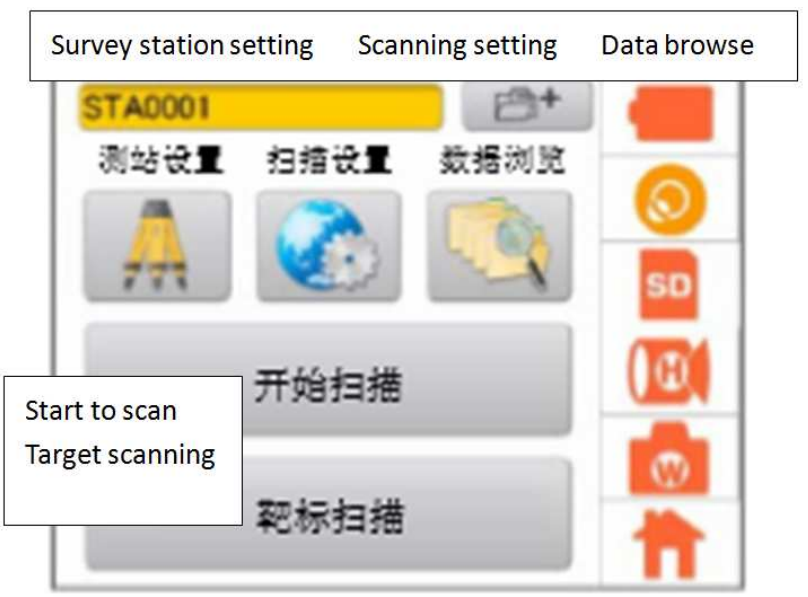

Figure 4. Leveling (2).

The electronic bubble appears on the display screen at that time [7]. A laser beam is shot from the bottom of the instrument after clicking the icon of '[i] . Loosen fastening screws on the tripod; adjust the instrument to make the centering point on the center mark; tighten fastening screws on the tripod [8]. Do not screw the base while adjusting the instrument so as to prevent the bubble from deviation.

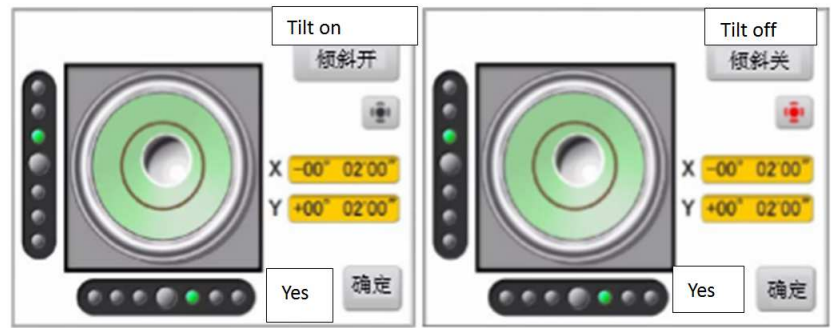

Figure 5. Leveling (3). 


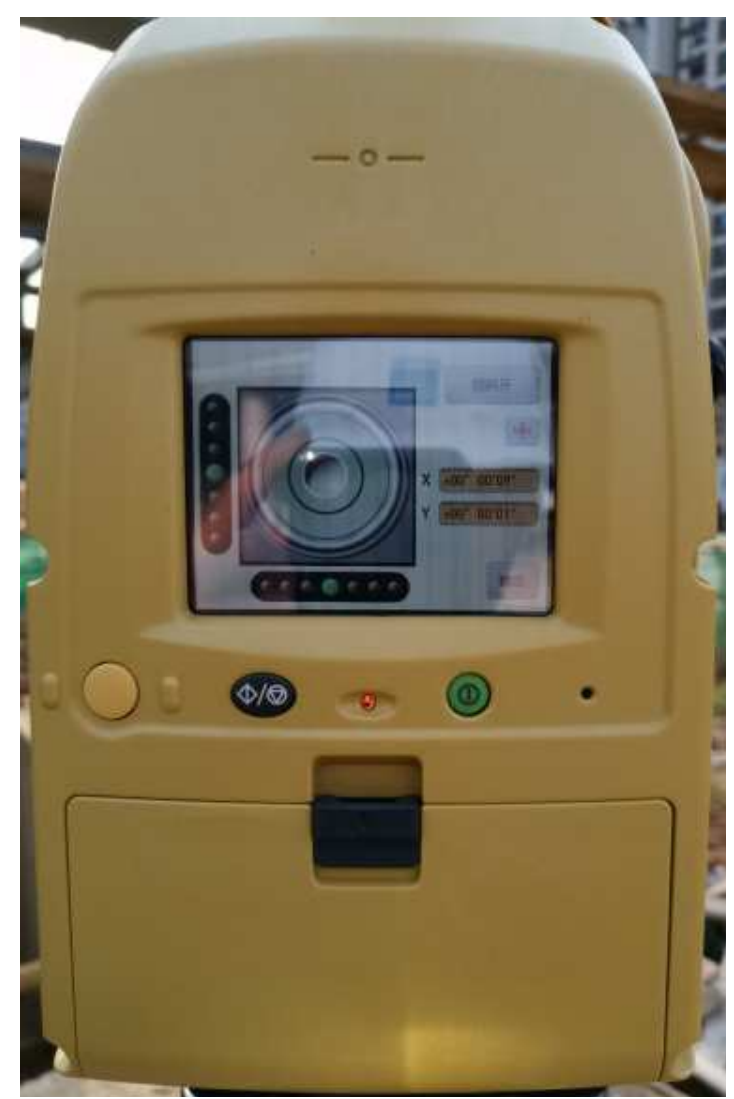

Figure 6. Leveling (4).

\subsection{Edit Coordinate Point File}

Import coordinate file or create CSV file (coordinate file).

\subsubsection{Import Coordinate File}

The equipment can identify the coordinate file in CSV format stored in SD card and import coordinate point name and coordinate value in the file into the memory as survey station point or back-sight point of scanning operation.

1) Click the icon of [Survey station setting]

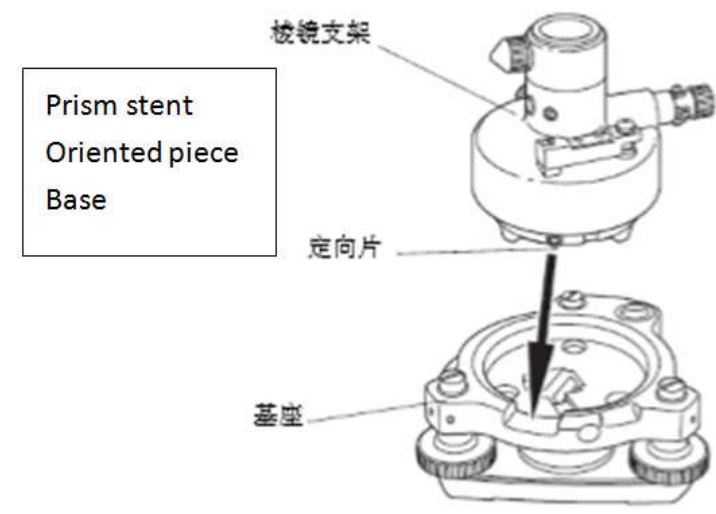

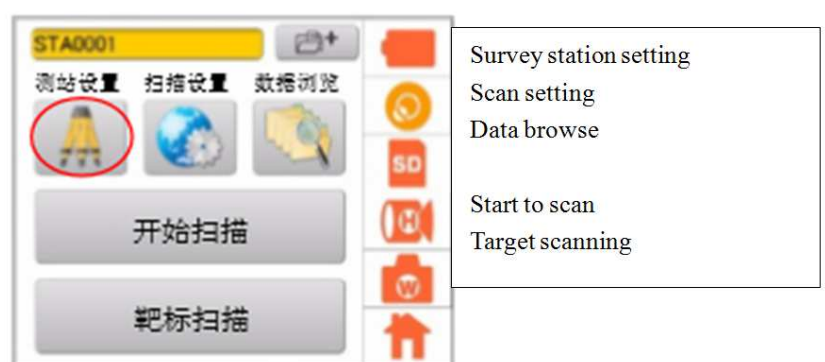

Figure 7. Edit Coordinate Point File (1).

2) Click the icon of [Survey station point name] or [back-sight point name]

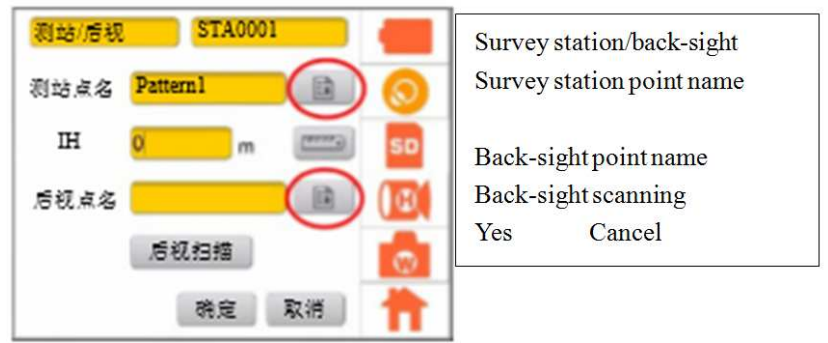

Figure 8. Edit Coordinate Point File (2).

3) Select the file in CSV format to be imported; click [Yes] to successfully import the selected file in CSV format.

\subsubsection{Create Coordinate Data}

In case coordinate data of the survey station point needs to be input, input data is unnecessary to be imported; the coordinate point can be directly clicked for edition [9].

\subsection{Install a Prism}

Align the oriented piece on the prism stent to the guide slot of the base; tighten fastening buttons on the base clockwise; install the prism; tighten fastening screws after installation so as to make the prism face to the $3 \mathrm{D}$ laser scanner [10].

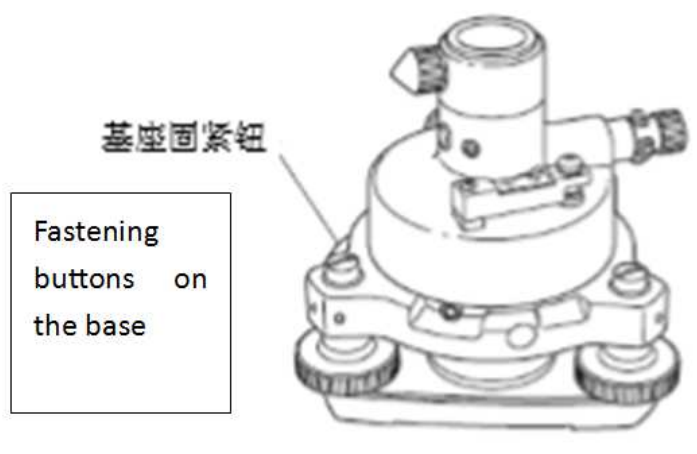

Figure 9. Install a Prism.

\subsection{Orientation and Coordinate Establishment}

Align the scanning lens of the 3D laser scanner to the target direction after installation of the prism; bring up the dot interface on the operation panel; align the cross cursor in the middle of the screen on the displayed image to the target or single prism center; automatically scan the target.

\subsection{Camera Setting}

The built-in camera in the instrument shall automatically 
obtain images of the scanned area and keep them at the time of scanning.

Image data in the whole scanned area is constituted by many pictures. As the scanned area has been expanded, the number of pictures shot has been increased as well [11]. Therefore, "wide-field camera" or "telephoto camera" shall be selected as required at the time of shooting before scanning [12].

\subsection{Scan Setting}

Preset resolution and user-defined resolution can be used for changing scan setting options and selecting the best scanning mode [13].

\subsection{D Scanning}

Obtain 3D data of scanned objects through 3D scanning.

Determine scanning groups and set relevant scanning parameters:

Rotate the instrument and roughly target the upper left corner of the scanned area after entering the scanned area setting interface; accurately select the upper left corner of the scanned area on the instrument display screen [14]; rotate the instrument and roughly target the upper right corner of the scanned area after checking the point location; accurately select the upper right corner of the scanned area on the display screen.

Scanning shall start; shooting shall appear on the display screen; the instrument shall automatically scan the scanned area after shooting [15].

\section{Advantages of 3D Laser Scanner}

(1) Large-scale scanned area and fast speed;

(2) Fully automatic scanning operation to reduce workload of survey crew;

(3) Overall scanning to reduce number of survey crew and time input;

(4) Direct generate models with scanned data through point cloud software so as to reduce time input of modeling.

\section{Conclusion}

3D laser scanner has been applied to China Building Science and Technology Museum Project, CSCEC Star of Optical Valley for measurement and remeasurement and been highly recognized by the Designer, the Employer and other parties in convenience, high efficiency and other aspects [16]. In consideration of high efficiency, convenience and bonding to BIM of 3D laser scanner in China Building Science and Technology Museum Project, can shorten the construction period and reduce the labor input [17], it is believed that 3D laser scanner will be the preferred measurement measure for special-shape curved curtain wall construction in the future and will be widely promoted and applied in the field and create greater value.

\section{References}

[1] Wang Mo. Application of Three-Dimensional Laser Scanning Technology in Surveying and Mapping of Ancient Buildings in the Imperial Palace [J]. Palace Museum Journal/m-. 2011 (06).

[2] Zhang Qifu, Sun Xianshen, Wang Li. Research on Precision Test Method of RIEGL VZ-400 Laser Scanner Based on Simple Six-section Method [J]. Geotechnical Investigation \& Surveying. 2011 (03).

[3] Wang Changhan, Xiang Zejun, Liu Jie. Application of 3D Laser Scanning Technology in 3D Reconstruction of Cultural Relics [J]. Urban Geotechnical Investigation \& Surveying. $2010(06)$.

[4] Zheng Dehua, Yue Dongjie, Yue Jianping. Building Point Cloud Registration Algorithm Based on Geometric Feature Constraint [J]. Acta Geodaetica et Cartographica Sinica. 2008 (04).

[5] Cheng Xiaojun, Guan Yunlan, Hu Shengli, et al. Research on Calibration of Additive and Multiplying Constants in Range Finding of HDS3000 Ground Laser Scanner. Collected Papers of Nanchang Optical Joint Annual Meeting of Five Provinces and One City and the Optical Joint Annual Meeting of 13 Provinces. 2008.

[6] Li Lu, Zhang Ailin. Application of 3D Scanning Combined with BIM Technology in Glass Curtain Wall Engineering [J]. Value Engineering. 2016 (08).

[7] Li Jian, Wang Zongmin, Ma Yurong, Tian Zhizhi. Research on Full-automatic High-Precision Splicing Method of Multi-Station Laser Point Cloud Data [J]. Geomatics and Information Science of Wuhan University. 2014 (09).

[8] Yangbin Lin, Cheng Wang, Jun Cheng, Bili Chen, Fukai Jia, Zhonggui Chen, Jonathan Li. Line segment extraction for large scale unorganized point clouds [J]. ISPRS Journal of Photogrammetry and Remote Sensin. 2015.

[9] Susanne Becker. Generation and application of rules for quality dependent fa?ade reconstruction [J]. ISPRS Journal of Photogrammetry and Remote Sensing. 2009 (6).

[10] Mai Feilong. Analysis Model and Application Research on Curtain Wall Engineering of Special-Shaped Building [D]. Shanghai Jiaotong University. 2013.

[11] Wang Daibing, Yang Hongyan, Xing Yafei, Wang Hankun, Li Jian. Application of BIM and 3D Laser Scanning Technology in Reverse Construction of Curtain Wall Project of Tianjin CHOW TAI FOOK Financial Center $[\mathrm{J}]$. Construction Technology. 2017 (23).

[12] Dinesh M, Ryosuke S. Auto_extraction of Urban Features from Vehicle_Borne Laser Data. 2002.

[13] He Xing. Construction Technology and Quality Control of Metal Panel Curtain Wall in Guangzhou International Sports Arena [D]. Xi'an University of Architecture and Technology. 2014.

[14] Yang Wenjun, Wan Limin, Ji kangdong, Cai Qingjun, Zhang Liang. Construction Technology of Large Hyperboloid Single-Cable Glass Curtain Wall in Dongguan Basketball Center [J]. Construction Technology. 2012 (02). 
[15] Xue Huazhu, Xu Keke. Research and Realization of 3D Laser Scanning in Building Model Construction [J]. Li Changchun, Journal of Henan Polytechnic University (Natural Science). $2008(02)$.

[16] Comparing pommes and naranjas. LAISERIN J. http://www.laiserin.com. 2002.
[17] H. L. Guo, Heng Li, Martin Skitmore. Life-Cycle Management of Construction Projects Based on Virtual Prototyping Technology. Journal of Management. 2010. 